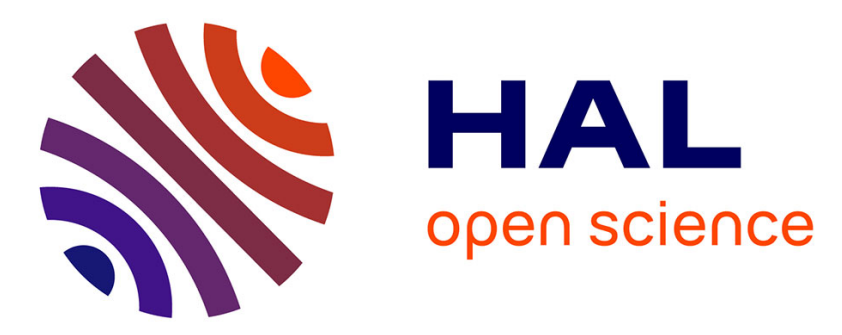

\title{
Study of structure and electrical conductivity in microemulsions : Evidence for percolation mechanism and phase inversion
}

\author{
M. Lagües, R. Ober, C. Taupin
}

\section{- To cite this version:}

M. Lagües, R. Ober, C. Taupin. Study of structure and electrical conductivity in microemulsions: Evidence for percolation mechanism and phase inversion. Journal de Physique Lettres, 1978, 39 (24), pp.487-491. 10.1051/jphyslet:019780039024048700 . jpa-00231552

HAL Id: jpa-00231552

https://hal.science/jpa-00231552

Submitted on 1 Jan 1978

HAL is a multi-disciplinary open access archive for the deposit and dissemination of scientific research documents, whether they are published or not. The documents may come from teaching and research institutions in France or abroad, or from public or private research centers.
L'archive ouverte pluridisciplinaire HAL, est destinée au dépôt et à la diffusion de documents scientifiques de niveau recherche, publiés ou non, émanant des établissements d'enseignement et de recherche français ou étrangers, des laboratoires publics ou privés. 


\title{
STUDY OF STRUCTURE AND ELECTRICAL CONDUCTIVITY IN MICROEMULSIONS : EVIDENCE FOR PERCOLATION MECHANISM AND PHASE INVERSION
}

\author{
M. LAGÜES, R. OBER and C. TAUPIN \\ Laboratoire de Physique de la Matière Condensée (*), Collège de France, \\ 11, place Marcelin-Berthelot, 75231 Paris Cedex 05, France
}

(Reçu le 17 août 1978, révisé le 20 octobre 1978, accepté le 30 octobre 1978)

\begin{abstract}
Résumé. - La structure et la conductibilité électrique de microémulsions ont été étudiées dans la région concentrée. Dans la zone où la structure de la gouttelette reste inchangée par la concentration, la variation de la conductivité sur 5 décades s'interprète bien par un modèle de percolation. Au-delà de l'empilement aléatoire compact des gouttelettes, la structure s'inverse (eau/huile $\rightarrow$ huile/eau) et la conductivité change de mécanisme.
\end{abstract}

\begin{abstract}
We present a study of structure and electrical conductivity in concentrated microemulsions. In the concentration range where the structure of the droplet is unchanged, the giant changes of the conductivity over 5 decades are interpreted in terms of a percolation model. Above the random packing fraction density the structure is inverted $(w / o \rightarrow o / w)$. The mechanism of conductivity is modified.
\end{abstract}

1. Introduction. - Microemulsions are transparent liquid dispersions of two immiscible liquids (i.e. water and oil). In a wide range of composition they are composed of minute droplets surrounded by a film of amphiphilic molecules. Many questions remain about the domain where the ratio of water to oil is high (concentrated region).

Several studies of these systems of both fundamental and industrial importance, have appeared recently [1-5].

In a recent paper [6], we described the structure of the elementary droplet of microemulsions containing water, cyclohexane, sodium dodecylsulphate and 1-pentanol. It has been shown that the structure of the droplet may be kept constant when the water concentration is varied.

In the present paper, we present a structural study and measurements of the electrical conductivity of microemulsions as a function of water concentration in the high concentration range. The variable contrast method in neutron scattering is used as a tool to detect the changes of the structure.

$\left(^{*}\right)$ E.R.A. 542 du Centre National de la Recherche Scientifique.
2. Neutron scattering study. - Let us recall that, in the static approximation and for particles with spherical symmetry, the expression for the coherent scattering cross-section separates into two terms, one reflecting the internal structure of the particle and the other the correlations between particles so that, if the contrast is varied at constant concentration, information on the internal structure is obtained.

The variation of the intensity $i$, extrapolated to zero value of the momentum transfer $q$, is proportional to the contrast factor $K$ of the particle which can be written :

$$
i_{(q=0)} \propto K=\left[\Sigma_{i} v_{i}\left(\rho_{i}-\rho_{\mathrm{o}}\right) / v\right]^{2}
$$

$\rho_{\mathrm{o}}$ is the mean scattering length of the solvent and the particle is divided into homogeneous parts of volume $v_{i}$ with mean scattering length $\rho_{i}$.

From expression (1) it is clear that the plot of $\sqrt{i_{(q=0)}}$ as a function of $\rho_{\mathrm{o}}$ is linear for an homodisperse sQlution of particles [7]. The value of $\rho_{\mathrm{o}}$ corresponding to the minimum (which is zero for perfectly homodisperse particles) gives a relationship between the $v_{i}$ 's and $\rho_{i}$ 's.

In the case of dilute microemulsions, a model of spherical droplets with two densities corresponding 
to the water core and to the interfacial film has shown to be valid [6]. If $R_{\mathrm{w}}$ and $R_{\mathrm{o}}$ are the radii corresponding to the boundaries of the interfacial film, respectively, with the water and the oily phase, the condition of zero contrast reduces to a particularly simple expression :

$$
\left(\frac{R_{\mathrm{w}}}{R_{\mathrm{o}}}\right)^{3}=\frac{\rho_{\mathrm{o}}-\rho_{\mathrm{I}}}{\rho_{\mathrm{w}}-\rho_{\mathrm{l}}}
$$

where $\rho_{\mathrm{w}}, \rho_{\mathrm{l}}, \rho_{\mathrm{o}}$ are the scattering length densities of, respectively

- the water core,

- the protonated part of the interfacial film (soap and excess pentanol as shown in [6]),

- the continuous phase which contains cyclohexane, pentanol and a small amount of water.

With this notation, (2) remains valid even in the case of an inverted (oil/water) system (Fig. 1).

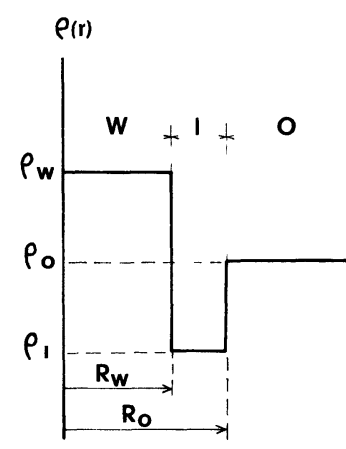

a

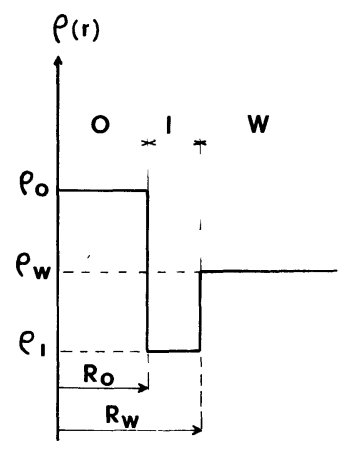

b
FIG. 1. - Schematic representation of the contrast used in small angle neutron scattering experiments. Indexes w. o. I. refer respectively to oil, water and interface. a) correspond to usual w/o microdroplet and $b$ ) to an inverse (o/w) system.

Several points should be noted :

a) The range of variation of $\rho$ (in our case $\rho_{\mathrm{o}}$ or $\rho_{\mathrm{w}}$ ) is usually limited by chemical considerations and it may not be possible to reach the point of zero contrast (Figs. $2 \mathrm{I} b$ and $2 \mathrm{II} a$ ). In this case, the extrapolation of the curve $\sqrt{i(\rho)}$ still gives the zero contrast value of $\rho$, but with a poorer precision.

b) The depth of the minimum is related to the polydispersity of the system ; a quantitative correlation is difficult to find but qualitative information may be obtained.

c) $R_{\mathrm{o}}$ and $\rho_{\mathrm{o}}$ in equation (2) are both functions of the partition coefficient of the alcohol, respectively, between the continuous phase and the interfacial film. As these quantities have to be consistent with the total alcohol amount, both $R_{\mathrm{w}} / R_{\mathrm{o}}$ and the excess of pentanol are determined by equation (2).

d) When the condition of zero contrast is fulfilled, the value of the scattering length of the external phase is necessarily intermediate between the two other

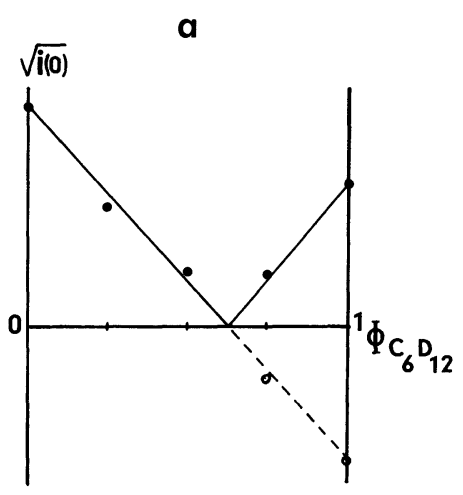

b

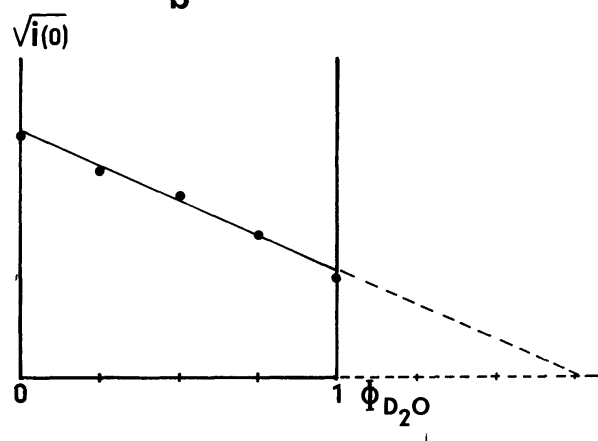

I

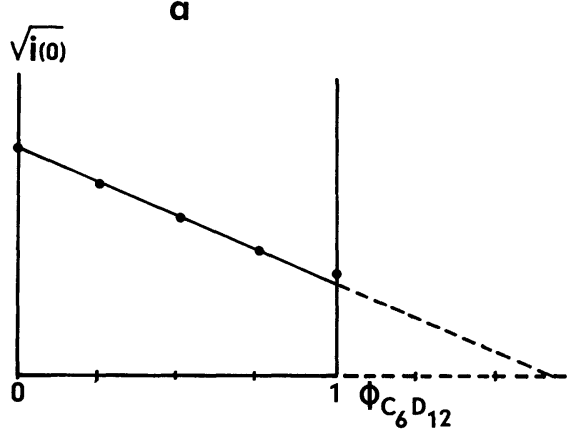

b

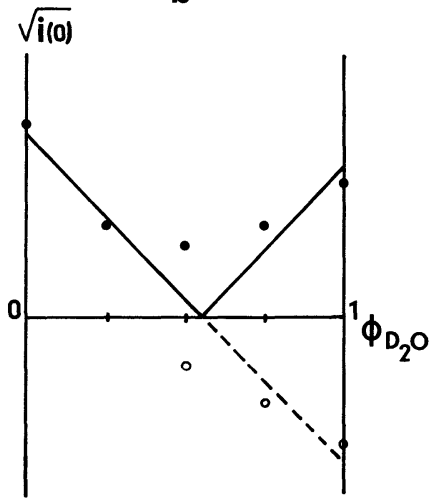

II

Fig. 2. - The square root of the extrapolated zero angle scattering intensity is plotted versus the volume fraction $\varphi$ of deuterated compound : a) $\mathrm{C}_{6} \mathrm{H}_{12}-\mathrm{C}_{6} \mathrm{D}_{12}$ mixture ; b) $\mathrm{H}_{2} \mathrm{O}-\mathrm{D}_{2} \mathrm{O}$ mixture ; I $\varphi_{w}=0.017 ;$ II $\varphi_{\mathrm{w}}=0.38$.

values. This provides an easy way, either to determine the external phase or to detect a phase inversion.

Figure 2 I shows the experimental results obtained for a diluted microemulsion (labeled as type B in [6] : 
water to soap ratio of 2.5 in weight) with two different variable contrasts. The two types of contrast $\mathrm{a}$ and $\mathrm{b}$ give the same value for $R_{\mathrm{o}} / R_{\mathrm{w}}$.

We used this method to check :

- any change of the structure of the elementary droplet,

- an eventual phase inversion of the structure, due to concentration.

In fact, concentration is not easily defined in microemulsions since several volumes for the droplet may be considered :

- water volume,

- chemical volume corresponding to water + soap + excess alcohol as shown in [6],

- hydrodynamical volume.

We will define as $\varphi_{\mathrm{w}}$ the volume fraction of water in the microemulsion. Following [6] the volume fraction occupied by the droplets (water + chemical interface) is given by

$$
C=\varphi_{\mathrm{w}}\left(1+x+y_{\mathrm{E}}\right)
$$

where $x$ is the volume ratio of soap to water and $y_{\mathrm{E}}$ the volume ratio of excess alcohol to water; for $B$ type microemulsions, $x=0.345$ and $y_{\mathrm{E}}$ was evaluated between 0.35 and 0.5 depending on the method of measurement.

Table I shows that up to $\varphi_{\mathrm{w}}$ equal to 0.30 the structure of the droplet is unchanged.

\section{TABLE I}

Results of the variable contrast study for different water volume fraction $\varphi_{\mathrm{w}} . R_{\mathrm{w}}$ and $R_{\mathrm{o}}$ are the radii limiting the interfacial film. $y_{\mathrm{E}}$ is the ratio of excess alcohol to water (in volume) in the droplet as determined in [6].

$\begin{array}{lllllll}\varphi_{\mathrm{w}} & 0.0166 & 0.076 & 0.103 & 0.134 & 0.244 & 0.300 \\ \frac{R_{\mathrm{o}}}{R_{\mathrm{w}}} & 1.18 & 1.17 & 1.17 & 1.17 & 1.17 & 1.17 \\ y_{\mathrm{E}} & 0.38 & 0.34 & 0.35 & 0.34 & 0.33 & 0.33\end{array}$

Figure 2 II shows that for volume fractions $\varphi_{\mathrm{w}}$ greater than 0.34 , the aspect of the variable contrast curve is completely different : with the $\left(\mathrm{C}_{6} \mathrm{H}_{12}-\mathrm{C}_{6} \mathrm{D}_{12}\right)$ mixture, one does not reach a minimum. If $\left(\mathrm{C}_{6} \mathrm{D}_{12}\right.$, $\mathrm{H}_{2} \mathrm{O}-\mathrm{D}_{2} \mathrm{O}$ ) contrast is used it is possible to reach a minimum. The use of equation (2) shows that for these high concentrations the external phase is the aqueous phase, revealing a phase inversion. This phase inversion in microemulsions has been frequently supposed to occur in these systems but without definite experimental proof.

It has to be noted that the variable contrast curve of figure 2 II indicates a higher polydispersity of the particles in the inverted phase. Some polydispersity also appears just before the inversion point.
3. Electrical conductivity. - The measurements were performed at $20 \pm 0.1^{\circ} \mathrm{C}$ using a $600 \mathrm{~Hz}$ a.c. current to avoid electrophoresis. As the conductivity is sensitive to the composition, its value was measured during the titration of the microemulsion by alcohol. A maximum in the value of the conductivity is observed at the clearing point. This maximum was considered as the conductivity of the microemulsion. This experimental procedure gives a reproducibility of about 2 per cent.

Figure 3 presents the variation of the conductivity of the type B microemulsion as a function of water concentration.

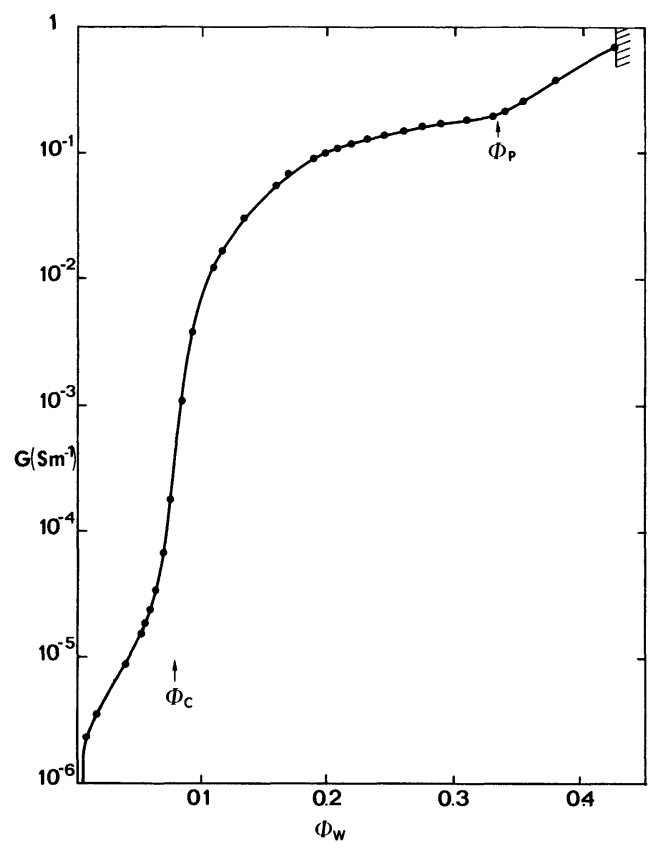

FIG. 3. - Variation of the conductivity of a microemulsion (logarithmic plot) versus water concentration.

It increases steeply (two orders of magnitude) between $\varphi_{\mathrm{w}}=0.06$ and $\varphi_{\mathrm{w}}=0.09$ : in this range of concentration the droplets fill around $15 \%$ of the space which is the theoretical value of the percolation threshold for hard spheres [8]. This led us to use percolation theory to describe our results.

Let us recall that the percolation concept applies to a random spatial distribution of identical objects : above a critical value $\varphi_{\mathrm{c}}$ of the volumic concentration of objects, an infinite path appears through them. Various physical properties of the system, for instance the mean conductivity, vary steeply around the percolation threshold $\varphi_{\mathrm{c}}$, obeying universal laws independent of the detailed physical properties of the system. In the following, we analyse successively the concentration dependence of the conductivity above and below this threshold.

1) In the region above and in the vicinity of the percolation threshold, numerical simulation and theory predict a power law [8] :

$$
G\left(\varphi_{\mathrm{w}}\right)=G_{0}\left(\varphi_{\mathrm{w}}-\varphi_{\mathrm{c}}\right)^{t}
$$


where $\varphi_{\mathrm{c}}$ is the percolation threshold and the exponent $t$ is around 1.6. In order to determine $\varphi_{\mathrm{c}}$ and $t$ independently, we plot $G^{1 / x}$ versus $\varphi_{\mathrm{w}}$ for $x=1.4,1.5$, 1.6, 1.7 (Fig. 4). It appears that for $x=1.5$ and 1.6, the plot is satisfactorily linear. We thus propose the value

$$
t=1.55 \pm 0.1
$$

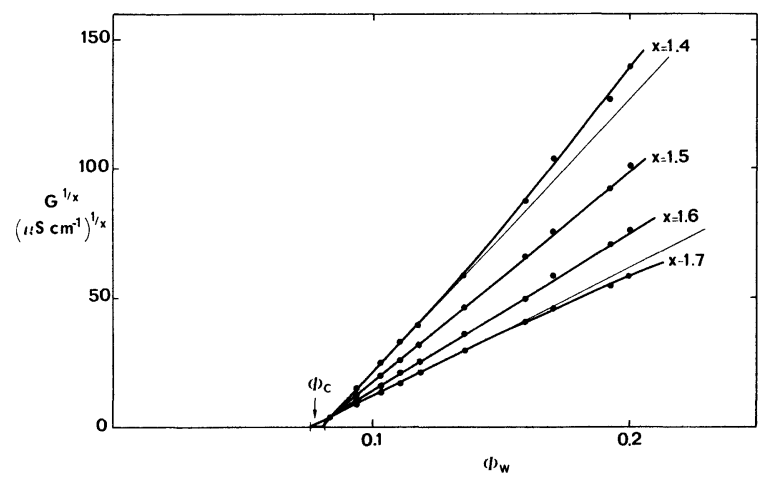

Fig. 4. - Determination of the exponent and the threshold value $\varphi_{c}$ of the power law of eq. (4) for the conductivity $G$ above the percolation threshold $\varphi . G^{1 / \AA}$ is plotted for various $x$ as a function of $\varphi_{\mathrm{w}}$.

The water concentration $\varphi_{\mathrm{c}}$ at the threshold is obtained by extrapolation to $G^{1 / x}=0$ :

$$
\varphi_{\mathrm{c}}=0.078 \pm 0.002 \text {. }
$$

The part of space filled by the droplets is, in fact, due to the water and the corresponding volume of the interphase.

At the threshold, the volumic fraction $C_{\mathrm{c}}$ of space filled by the droplets is deduced from eq. (3) :

$$
C_{\mathrm{c}}=0.14 \pm 0.01
$$

if $y_{\mathrm{E}}=0.5 \pm 0.1$.

This is in agreement with the theoretical value of 0.148 for a f.c.c. packing [8].

The agreement for both $t$ and $C_{\mathrm{c}}$ with the values predicted by percolation calculations confirms that conductivity is controlled by percolation of the droplets : above $\varphi_{\mathrm{c}}$ there always exists an infinite path through the droplets, much more conductive than the continuous phase.

At $\varphi_{\mathrm{P}}=0.332$, which corresponds to the structure modification observed by neutron scattering, the curve of figure 3 exhibits a break and the conductivity increases more rapidly. The part of space $C_{\mathrm{P}}$ filled at $\varphi_{\mathrm{P}}$ by the droplets is :

$$
C_{\mathrm{P}}=0.630
$$

which should be compared with the random packing volume fraction for hard spheres : 0.637 [9].

The conductivity for this concentration is

$$
G_{\mathrm{P}}=0.195 \mathrm{Sm}^{-1} \text {. }
$$

This value may be compared to the value of the conductivity of a $\mathrm{NaCl}$ solution containing the same number of ions : $4.5 \mathrm{Sm}^{-1}$. This relatively low value of the conductivity $G_{\mathrm{P}}$, may be understood if the conduction in the interfacial films is the principal conduction mechanism for $\varphi_{\mathrm{c}}<\varphi_{\mathrm{w}}<\varphi_{\mathrm{p}}$. Indeed, neutron scattering results insure that in this range of concentration the aqueous cores of the droplets remain completely disconnected. On the contrary, the increase of conductivity when $\varphi_{w}>\varphi_{p}$ could arise from a progressive connection of the aqueous phase, related to the structural inversion which is observed by neutron scattering.

2) The phase transition theory predicts that the conductivity of percolating conducting sites which are fixed in an insulating medium seems to diverge below the percolation threshold :

$$
G \sim\left(\varphi_{\mathrm{c}}-\varphi_{\mathrm{w}}\right)^{-S}
$$

Very few predictions are available for $S$ : the effective medium theory gives $S=1$ and lattice simulations suggest that $S=0.7$ in three dimensions [10].

We must point out that in our case of a liquid suspension, the mobility of the conducting particles may completely transform the theoretical predictions.

Figure 5 presents a $\log -\log$ plot of our conductivity results versus $\left(\varphi_{\mathrm{c}}-\varphi_{\mathrm{w}}\right)$.

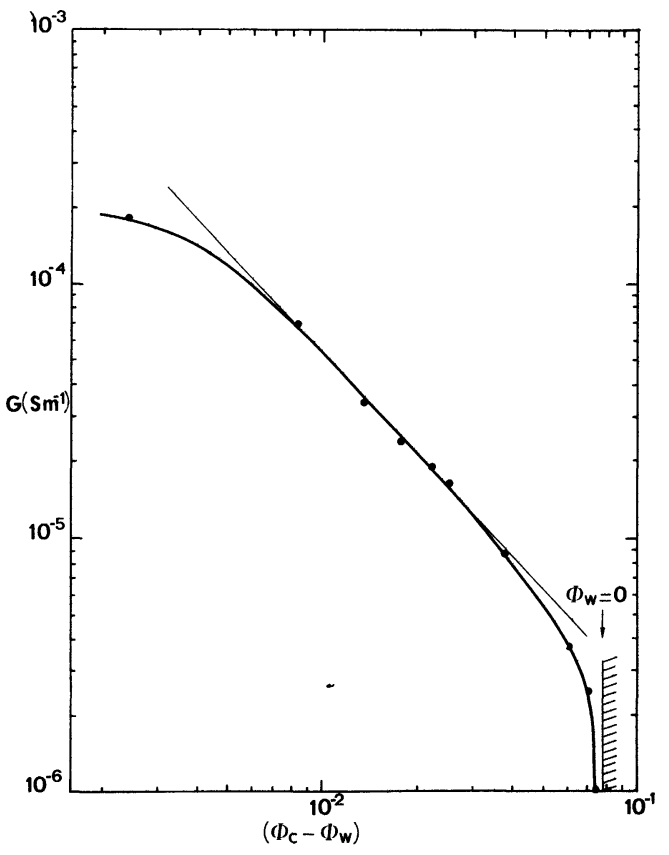

FIG. 5. - Log-Log plot of the conductivity $G$ versus the volume fraction $\left(\varphi_{\mathrm{c}}-\varphi_{\mathrm{w}}\right)$ below $\varphi_{\mathrm{c}}$ in order to check eq. (5).

When $0.008<\left(\varphi_{c}-\varphi_{w}\right)<0.02$ the plot is linear and the experimental exponent $S$ is found to be 1.32 .

By contrast, the conductivity for very dilute microemulsions $\left(\left(\varphi_{c}-\varphi_{w}\right)>0.02\right)$ varies approximately as $\varphi_{w}$. This conduction, much greater than that of 
the continuous phase, could be due to the nonneutrality of the mobile droplets.

As the mobility of the droplets has been previously determined [6], it is possible to evaluate the mean charge by droplet which is found of the order of one electronic charge (one ion for 600 molecules of soap in the interfacial film).

The variation of the conductivity below the threshold seems thus to involve the mobility of the droplets; this could explain the departure of the $S$ expo- nent from the theoretical predictions valid only for fixed conducting elements in an insulating medium.

Acknowledgments. - We are indebted to P. G. de Gennes, G. Giraud and E. Guyon for useful informations. We thank one of the referees for his suggestion to include the effective medium result for $S$.

This work was supported by a grant from the Délégation Générale à la Recherche Scientifique et Technique.

\section{References}

[1] Prince, L. M., Surfactant Science Series, édité par K. J. Lissant (Dekker, New York) 1976, Chap. 3. Vol. 6. Part I.

[2] Shah, D. O., Walker, R. D., Hsich. W. C.. Shah, N. J., Dwidedi, S., Nelander. J., Pepinshi, R., Deamer, D. W., S.P.E. of AIME, paper number SPE 5815 (1976).

[3] Friberg, S., Lapczynska, I. et Gillberg, G., J. Colloid Interface Sci. 56 (1976) 19.

[4] Cayias, J. L., Schechter, R. S.. Wade, W. H., J. Colloid Interface Sci. 59 (1971) 31-33.
[5] Biais, J., Lalanne. P.. Clin. B.. Bellocq. A. M.. Lemanceau, B., J. Chim. Phys. 74 (1977) 1197.

[6] Dvolaitzky, M.. Guyot, M.. Lagües. M.. Le Pesant. J. P., Ober, R., Sauterey, C.. Taupin. C.. to appear in $J$. Chem. Phys.

[7] Stuhrmann, H. B., J. Appl. Crystallogr. 7 (1974) 173.

[8] Kirkpatrick, S.. Rev. Mod. Phys. 45 (1973) 574

[9] FinNey, J. L., Nature 266 (1977) 309.

[10] Straley, J. P., Phys. Rev. 15B (1977) 5733. 